\title{
Impact of Fermi-LAT and AMS-02 results on cosmic-ray astrophysics
}

\author{
Charles D. Dermer ${ }^{\mathrm{a}}$ \\ Space Science Division, US Naval Research Laboratory, Washington, DC, USA
}

\begin{abstract}
This article reviews a few topics relevant to Galactic cosmic-ray astrophysics, focusing on the recent AMS-02 data release and Fermi Large Area Telescope data on the diffuse Galactic $\gamma$-ray emissivity. Calculations are made of the diffuse cosmic-ray induced $p+p \rightarrow \pi^{0} \rightarrow 2 \gamma$ spectra, normalized to the AMS-02 cosmic-ray proton spectrum at $\approx 10-100 \mathrm{GV}$, with and without a hardening in the cosmic-ray proton spectrum at rigidities $R \gtrsim 300 \mathrm{GV}$. A single power-law momentum "shock" spectrum for the local interstellar medium cosmic-ray proton spectrum cannot be ruled out from the $\gamma$-ray emissivity data alone without considering the additional contribution of electron bremsstrahlung. Metallicity corrections are discussed, and a maximal range of nuclear enhancement factors from 1.52 to 1.92 is estimated. Origins of the $300 \mathrm{GV}$ cosmic-ray proton and $\alpha$-particle hardening are discussed.
\end{abstract}

\section{Introduction}

On the way to writing this proceedings article for the $2^{\text {nd }}$ SUGAR (Searching for the sources of galactic cosmic rays) Workshop, held in the Département de Physique nucléaire et corpusculaire at Université Genève on 21-23 January 2015, the Alpha Magnetic Spectrometer Collaboration held a significant data release on its "AMS days at CERN," which took place 15-17 April 2015 [1]. Since new data is bound to make old theory more interesting, I've redirected my thoughts towards this latest high-quality cosmic-ray data.

The major results presented at the AMS days were the $\bar{p} / p$ ratio from kinetic energies $T_{p} \lesssim 1 \mathrm{GeV}$ to $T_{p} \approx$ $350 \mathrm{GeV}$, and the cosmic-ray proton and $\alpha$ particle spectra for rigidities $10 \mathrm{GV} \lesssim R \lesssim 1500 \mathrm{GV}$ (p) and $10 \mathrm{GV} \lesssim R \lesssim 2500 \mathrm{GV}(\alpha)$. Combining these results with the previous AMS-02 results on the positron fraction [2] and the cosmic-ray electron and positron fluxes [3], in addition of course to the vast wealth of cosmic-ray data [4], gives an unprecedentedly large and accurate data set for studying cosmic-ray physics.

In the most recent AMS-02 data [1], the $\bar{p} / p$ ratio is reported to be roughly constant for $10 \mathrm{GeV} \lesssim$ $T_{p} \lesssim 350 \mathrm{GeV}$ with a value of $\approx 2 \times 10^{-4}$. The measured $\bar{p} / p$ ratio is claimed to be a factor of $2-3 \times$ larger than predicted by secondary cosmic-ray production models constrained by, for example, the positron fraction $\mathrm{e}^{+} /\left(\mathrm{e}^{+}+\mathrm{e}^{-}\right)$and the $\mathrm{B} / \mathrm{C}$ ratio [5]. Given the associated rising positron fraction [2] at $\gtrsim 10 \mathrm{GeV}$, which is often explained as due to additional sources like pulsars, and not from enhanced cosmic-ray induced production, one may ask whether there is a consistent explanation for the overabundant $\bar{p}$ and the enhanced positron fraction.

AMS-02 confirms, though at a higher value of break rigidity, the hardening in the cosmic-ray proton and $\alpha$ spectrum [6] reported from PAMELA results [7]. The

a e-mail: charles.dermer@nrl.navy.mil hardening appears in the AMS-02 $\mathrm{p}$ and $\alpha$ spectra at $\approx 300-400 \mathrm{GV}$, compared to $\approx 240 \mathrm{GV}$ for cosmic-ray $\mathrm{p}$ and $\alpha$ spectra in PAMELA data [8]. Nevertheless, as was remarked more than once at this workshop, there must be a hardening between $\approx 100 \mathrm{GeV} /$ nuc and the knee, and it is great that AMS-02 has provided more precise details (which improves upon the preliminary spectra released during the 2013 ICRC [1]).

Rather than repeat the points made in my presentation, which is available at the conference website ${ }^{1}$ and reviews some of the work found in my previous conference papers $[9,10]$, it seems more useful to examine a few of the implications of the AMS-02 data in view of the goals of this meeting.

First we look at the cosmic-ray proton and $\alpha$-particle spectra from low energies to high energies, which represents the minimum local interstellar medium cosmic-ray proton $(\alpha)$ (LISMCRp $(\alpha)$ ) spectrum from which to calculate a minimum $\gamma$-ray emissivity.

Second, we make some calculations of galactic emissivity from the cosmic-ray proton data, using a simple nuclear enhancement factor $k_{n u c}$ to correct for metals. Even with $k_{n u c}=2$, a power-law momentum spectrum is allowed, so a contribution of bremsstrahlung $\gamma$ rays is required to infer a low-energy break in the LISMCRp spectrum from the Fermi-LAT $\gamma$-ray data. A brief discussion of metallicity $(Z>2, A>4)$ corrections, including cosmic-ray and ISM metals, is given. We consider whether the breaks in the spectra of cosmicray protons and ions are a consequence of propagation effects, multiple injection sources with different spectral indices, or due to hardening produced by nuclear collisions which preferentially depletes the low-energy particles that traverse larger grammages.

\footnotetext{
${ }^{1}$ https://indico.cern.ch/event/332221/session/5/ contribution/38
}

This is an Open Access article distributed under the terms of the Creative Commons Attribution License 4.0, which permits unrestricted use, distribution, and reproduction in any medium, provided the original work is properly cited. 

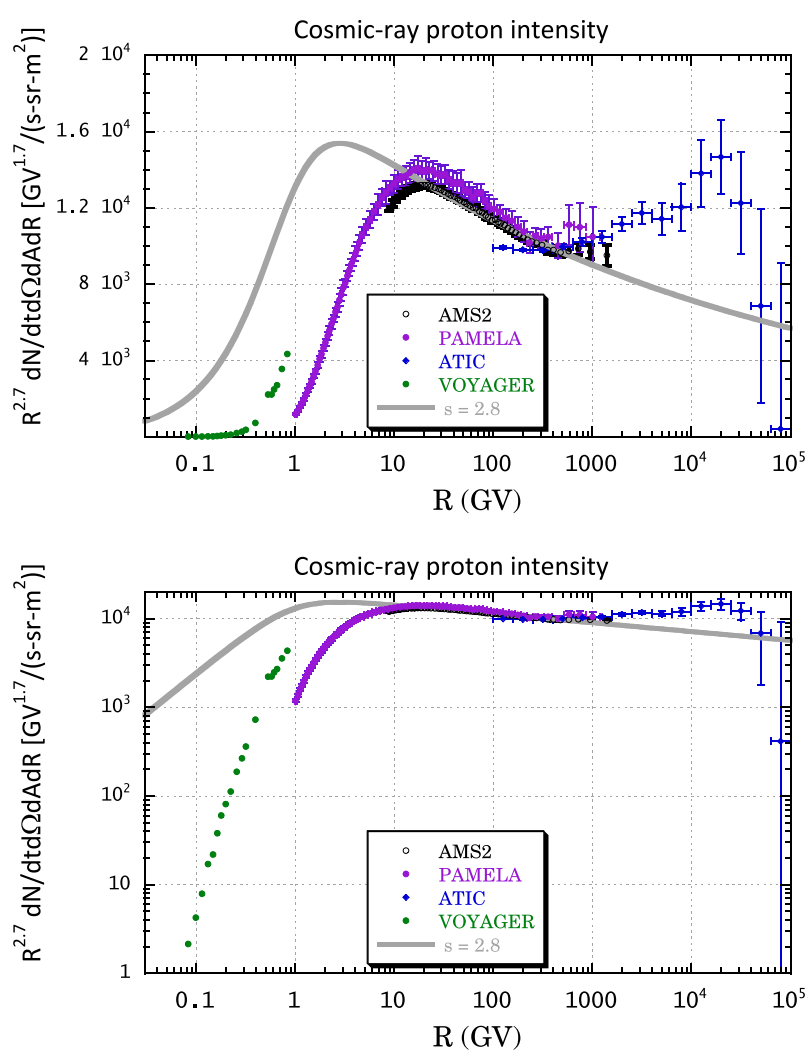

Figure 1. Data, as labeled, are from ATIC [11], PAMELA [7], AMS-02 [1], and Voyager 1 [12], expressed in the form $R^{2.7} \times$ particle number intensity. Smooth curves show a powerlaw proton flux in momentum with index $s=2.8$ in the same representation. Upper and lower panels show the fluxes on a linear and logarithmic scale, respectively.

\section{Cosmic rays and $\gamma$ rays}

\subsection{Cosmic-ray $p$ and $\alpha$ spectra}

In Fig. 1, we construct a plot of the cosmic-ray proton intensity (or flux) from 4 sets of data (see [4]) measured at or near Earth and at $\sim 122$ AU (Voyager 1). The highest energy data are from the ATIC (Advanced Thin Ionization Calorimeter)-2 balloon campaign during 2002-2003 [11]. Also shown are PAMELA (Payload for AntiMatter Exploration and Light-nuclei Astrophysics) data [7], the recently reported AMS-02 data [1], and Voyager 1 data [12]. One thing about which we can be quite certain is that due to Solar modulation of the cosmic ray, the LISMCRp spectrum will be above the level of the data. Though it could be that the Voyager 1 has sampled the "true" LISMCRp spectrum, it is also possible that during the billions of years that the Solar wind has been generated, a much more complicated and extended magnetic structure around the Sun has formed. In any case, the data should provide a solid lower limit to the LISMCRp spectrum. Another thing is that AMS-02 and PAMELA differ in normalization by $\lesssim 10 \%$, and more like $\sim 5 \%$ at $10 \lesssim \mathrm{R}(\mathrm{GV}) \lesssim 100$, where modulation effects are believed to be small. These differences are less than uncertainties in nuclear cross sections [13].

Figure 1 is a plot of the LISMCRp flux multiplied by $R^{2.7}$, where $R \equiv P / Z$ is the particle rigidity,
$P=\sqrt{E^{2}-m^{2}}$ is its momentum, $E$ is its total energy, and $T=E-m$ is the particle kinetic energy. For protons, then,

$$
R^{2.7} \frac{d N}{d A d t d \Omega d R}=\frac{R^{3.7}}{E T_{p}^{2}(R)}\left[T_{p}^{2} \frac{d N}{d A d t d \Omega d T_{p}}\right] .
$$

The upper and lower panels show the measured cosmic-ray proton flux on a linear and logarithmic scale, respectively. There are several points to be made. First is that the flux, as shown in this representation multiplied by $R^{2.7}$, deviates barely by a factor of 2 and is remarkably close to a power law from $10 \lesssim R(\mathrm{GV}) \lesssim 10^{4}$. Second is that three experiments-ATIC, AMS-02, and PAMELA-now converge on a break rigidity for protons of $R \approx 300 \mathrm{GV}$, with the low-energy $(10 \lesssim R(\mathrm{GV}) \lesssim 300)$ slope equal to $\approx 2.8$, and the high-energy $\left(300 \lesssim R(\mathrm{GV}) \lesssim 10^{4}\right)$ slope $\approx 2.6$, based primarily on the ATIC data (the high-energy extension of the AMS-02 data suggests that the hardening is less, perhaps to a slope of $\approx 2.7$ ).

\subsection{The cosmic-ray spectrum in the local interstellar medium}

What, then, is the LISMCRp spectrum and how is it made? The prevailing theory is that Galactic cosmic rays are accelerated by processes taking place in SNR shocks as the expanding supernova ejecta overtakes and sweeps up material in the surrounding medium [14]. This theory has been taught so much at school that to even question the SNR origin of Galactic Cosmic Rays at this stage is treated as heresy. ${ }^{2}$ In any event, this theory has received strong support from Fermi-LAT and AGILE observations of the $\pi^{0} \rightarrow 2 \gamma$ feature in IC 443 and W44 [15], as well as in the spectrum of a 3rd Fermi-LAT SNR, W51C, reported at this workshop by Dr. Jogler.

Although the $\pi^{0} \rightarrow 2 \gamma$ decay hardening in the lowenergy $\gamma$-ray spectrum below several hundred $\mathrm{MeV}$ has now been reported, confirming innumerable predictions and calculations going back to Ginzburg \& Syravotskii and Hayakawa, some open questions remain. For example, the SNR $\gamma$-ray spectral indices can be soft (i.e., steeper than 2.5), and unusually low high-energy cutoffs in the $\gamma$-ray spectra are inferred from Fermi-LAT and groundbased $\gamma$-ray detectors. For example, W51C and W44 have cutoffs below $10 \mathrm{GeV}$, whereas Cas A has a break near $1 \mathrm{TeV}$ and RX J1713.7-2942 has a break at $\approx 10 \mathrm{TeV}$. If the $\gamma$-ray emission is due to cosmic rays accelerated to the knee $(\approx 3 \mathrm{PeV}), \gamma$ rays at a factor of 10 less energy $(\approx 300 \mathrm{TeV})$, are expected, and no photons near that high are detected. (The highest energy photons, at $\approx 100 \mathrm{TeV}$, are observed in the steeply declining part of the RX J1713.7-3946 SNR spectrum [16].)

Putting these concerns to the side for the moment, we marvel instead that the cosmic-ray proton spectrum is so close to a power law, as expected in first-order testparticle Fermi acceration theory, as reviewed in [17]. One of the central results of this theory is that the distribution function of transmitted particles $f(p) \propto p^{-3 r /(r-1)}$, so that $d N / d p \propto p^{-A_{t p}}$, where the test-particle spectral index

$$
A_{t p}=\frac{2+r}{r-1},
$$

\footnotetext{
${ }^{2}$ Rather than burnt at the stake, one's proposal isn't funded.
} 
Here $r$ is the compression ratio which, for strong shocks in a medium with adiabatic index of $5 / 3$, appropriate to a nonrelativistic monoatomic gas, cannot exceed a value of $r=4$, implying a test-particle index of $A_{t p} \cong 2$ for a strong nonrelativistic shock.

If the injection spectrum of cosmic-ray protons or ions is a power-law in momentum or rigidity, then what is the form of the intensity $j_{s h}\left(T_{p}\right)=d N / d A d t d \Omega d T_{p}$ that enters into the calculations of $\gamma$-ray emissivity? Most simply, rigidity-dependent escape steepens the injection spectrum by the index $\delta$, so that the steady-state number spectrum $d N / d p \propto p^{-A_{t p}-\delta} \propto p^{-s}$, where $s=A_{t p}+\delta$. The intensity

$$
j_{s h}\left(T_{p}\right) \propto \beta\left(T_{p}\right) \frac{d N}{d T_{p}} \propto \beta\left(T_{p}\right)\left|\frac{d p}{d T_{p}}\right|\left(\frac{d N}{d p}\right) \propto p^{-s}
$$

[18]. In Fig. 1, a power-law momentum spectrum, normalized to the AMS-02 data at $10 \mathrm{GV} \lesssim R \lesssim 200 \mathrm{GV}$ with index $s=2.8$ is shown. It clearly disagrees with the Voyager 1 data, though as noted, this might not be a fatal concern if the Voyager 1 data does not yet sample the LISMCR spectrum.

In any case, however, one would have to be extremely naive to assume that a power-law momentum spectrum should describe the LISMCRp intensity. Besides diffusive escape from the target-rich Galactic disk, convective Galactic winds from the superpositions of the hot gases of $\mathrm{O} / \mathrm{B}$ stars and supernovae will cause a systematic expulsion of cosmic rays to the halo of the Galaxy. MHD turbulence in the Galactic magnetic field could cause systematic acceleration of cosmic rays during transport [19]. Although breaks may be expected, demonstrating such a break in the cosmic-ray proton spectrum has proven to be difficult, for reasons of (1) limitations on our knowledge of the secondary pion distributions in p-p collisions, and (2) metallicity corrections on secondary pion production $[18,20]$.

A crucial issue in the scenario that strong SNR shocks accelerate the Galactic cosmic rays should be noted. Compression ratios $r \rightarrow 4$ imply injection indices of cosmic rays near 2 . In theory the bulk of the cosmicray population is swept downstream and is confined to the remnant until late in the SNR's radiative evolution when the shock is not so strong, at which time the cosmic rays diffusively escape. The "real" injection index of cosmic rays into interstellar space may be somewhat softer, $\approx 2.1-2.2$. On the other hand, the highest energy particles will have escaped near the Sedov time. For these injection indices, the rigidity-dependent steepening would have to be by $\delta \approx 0.6-0.7$. This can be compared with analyses finding $\delta \approx 0.44 \pm 0.03$ and a source injection index of 2.34 [21] using the form $D(R)=D_{0} \beta\left(R / R_{0}\right)^{\delta}$ for the diffusion coefficient. PAMELA analysis finds $\delta \cong 0.4$ [22].

The secondary to primary ratios of cosmic-ray nuclei can also be modeled by an average escape grammage $\Lambda(R)$ through which cosmic-ray particles with rigidity $R$ pass [19]. Analysis of cosmic-ray ion composition often uses the empirical function $\Lambda(R)=\beta \Lambda_{0}\left(R / R_{0}\right)^{-a}$ for $R>R_{0}$ and $\Lambda(R)=\beta_{0} \Lambda_{0}$ for $R<R_{0}$, where $\beta=$ $p / E=Z R / \sqrt{Z^{2} R^{2}-m^{2}}$. When fit to the $\mathrm{B} / \mathrm{C}$ ratio, a typical parameter set fitting the data has $\Lambda_{0}=11.8 \mathrm{~g} / \mathrm{cm}^{-2}$ and $a=0.54$, and $R_{0}=4.9 \mathrm{GV} / \mathrm{c}$, with a rigidity index of 2.35. The value of $a$ essentially coincides with the value of $\delta$ in diffusion theory.

The value $\delta \approx 0.4$ is near the theoretically favored value of $\delta \approx 1 / 3$ for Kolmogorov turbulence, but observationally then requires a soft injection index $\approx 2.3-2.4$. In any case, cosmic rays must sample a wide range of environments, and it may be surprising that the cosmicray proton spectrum is as smooth as it is. Now we consider $\gamma$-ray constraints on deviations from a single momentum power law at the low- and high-energy parts of the Galactic cosmic-ray proton distribution.

\section{3. $\gamma$-ray constraints on cosmic rays}

We can ask if the power-law momentum spectrum shown in Fig. 1 can be ruled out by the $\gamma$-ray data. The expression $R^{2.7} d N / d t d \Omega d A d R=1.8 \times 10^{4}\left(R^{0.9} / E\right) \mathrm{GV}^{1.7} /(\mathrm{s}-$ $\mathrm{sr}-\mathrm{m}^{2}$ ) translates into a cosmic-ray proton flux

$$
j_{s h}\left(T_{p}\right)=1.8 R^{-2.8}\left(\mathrm{~s} \mathrm{sr} \mathrm{cm}^{2} \mathrm{GV}\right)^{-1} .
$$

(In comparison, I [23] used the expression $j_{s h}\left(T_{p}\right)=$ $2.2 E_{p}^{-2.75}\left(\mathrm{~s} \mathrm{sr} \mathrm{cm}^{2} \mathrm{GeV}\right)^{-1}$, with $E_{p}$ the total energy in $\mathrm{GeV}$, based on the cosmic-ray proton spectrum shown in Simpson's review [24].)

It is a simple matter to plug the proton flux given by Eq. (4) into a code that calculates the $\gamma$-ray emissivity per $\mathrm{H}$ atom due to pion-producing reactions of relativistic protons with protons at rest, and compare with the emissivity measured with the Fermi-LAT telescope. For this purpose, I use my original code [23], including the kludge between 3 and $7 \mathrm{GeV}$, which may be the source of some structure in the $\gamma$-ray production spectra. The improvements suggested [10] to properly characterize the different isobars according to their exclusive cross sections, which would mitigate the effects of the kludge, have not yet been fully implemented. We also make calculations using Kamae's model [27]. Notably, a nuclear enhancement factor $k_{n u c}=2.0$ is assumed.

Figure 2 shows calculations compared to Fermi-LAT data. The early Fermi-LAT emissivity study [25] uses data from 2008 August 4 to 2009 January 31 ( $\approx 6$ mo), whereas the 2012 study [26] uses 3 years of Fermi-LAT data. Besides the single momentum power-law shock spectrum, Eq. (4), we also consider a case where the shock spectrum hardens at $R_{b r}=300 \mathrm{GV}$ by 0.2 units, and a case where the spectrum nose dives below $2 \mathrm{GeV}$. Even with Pass 8 analysis and 10 years of Fermi-LAT data, it will be a big challenge to see a statistically significant hardening above $\approx 30 \mathrm{GeV}$. The two emissivities-with and without the spectral hardening at $R>300 \mathrm{GV}$ in the LISMCRp spectrum-differ by only a factor of 2 at $100 \mathrm{GeV}$ photon energies. Attempts to see the spectral hardening from Earth-limb analysis may be more successful, but limitations of our knowledge of secondary production cross sections can ultimately hinder analysis.

This simple comparison does not preclude the possibility that Eq. (4) could describe the LISMCRp spectrum, at least below $300 \mathrm{GV}$. In fact, the numerical fit using the simple shock power-law momentum spectrum, 


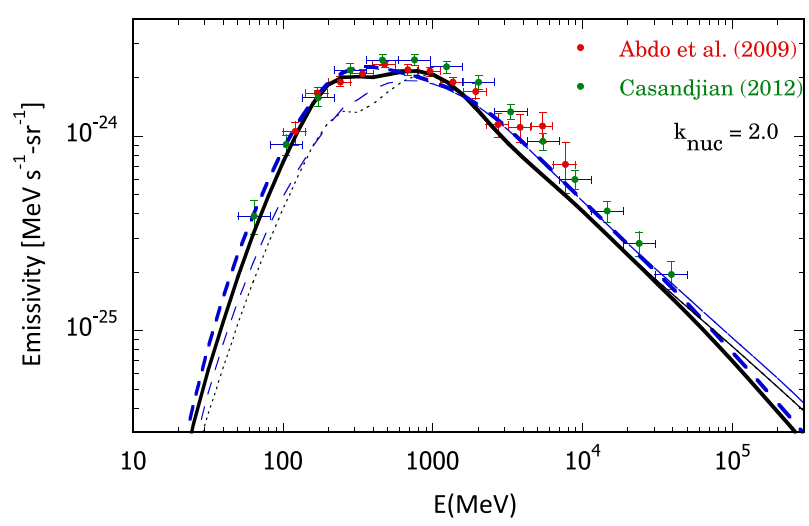

Figure 2. Fermi-LAT data shows early results of the Galactic $\gamma$-ray emissivity [25], and the emissivity from the 2012 analysis of Casandjian [26]. Calculations are for a proton momentum power-law shock spectrum given by Eq. (4) (thick), the same spectrum with a hardening by 0.2 units at $300 \mathrm{GeV}$ (thin solid), and the shock spectrum with a low-energy cutoff at $2 \mathrm{GeV}$ in the LISMCRp spectrum (thin dotted and dot-dashed); see Fig. 1. Two $p+p \rightarrow \pi^{0} \rightarrow 2 \gamma$ models are used, shown in black [23] and blue [27].

though going right through the low energy data, must allow some emission for bremsstrahlung from nonthermal electrons making radio synchrotron and for bremsstrahlung from the $\mathrm{e}^{+}$and $\mathrm{e}^{-}$made from the decay of $\pi^{ \pm}$. A full study must consider associated radio synchrotron emission [28], demodulation or reconstruction of the LISMCR $\mathrm{e}^{+} / \mathrm{e}^{-}$spectrum, and the inverse Compton emissions that are dependent on Galactic location [29,30]. A recent evaluation of $\gamma$-ray emissivity from the AMS-02 cosmic-ray proton and $\alpha$ data, including calculations of the associated neutrino fluxes, see [31]. Even so, emissions from leptons, because of their strong cooling, are expected to have softer spectra and contribute relatively less to the $\gamma$-ray emissivity at higher energies. It is not clear if the emissivity data is systematically $\sim 10-30 \%$ larger than predicted by cosmic-ray induced emissions when using $k_{n u c}=2$, and whether this is a reasonable value. This brings us to a reconsideration of the nuclear enhancement factor.

\subsection{Metallicity corrections}

The calculations shown in Fig. 2 make use of a simple multiplier for the effects of all nuclei other than protons and $\mathrm{H}$. This is the nuclear enhancement factor $k_{n u c}$, which was assigned a value of 2.0 in our calculations. Let us make a quick calculation of the range of values of $k_{n u c}$. The first thing to note is that in collision physics, $T_{n u c}$, the kinetic energy per nucleon is the quantity of interest. At the same values of $T_{n u c}$ we see that the accelerated particle distributions-the cosmic rays-have abundances of $\mathrm{H}, \mathrm{He}, \mathrm{CNO}$ and $\mathrm{Fe}$ in the ratio of 1:0.07:0.0044:0.0003 $[18,19]$. From Mori [32], drawing on the work of J.-P. Meyer [33], we take the composition of the target ISM material with relative abundances of $\mathrm{H}: \mathrm{He}: \mathrm{CNO}: \mathrm{NeMgSiS}: \mathrm{Fe}$ in the ratio 1:0.096:1.38e-3:2.11e-4:3.25e-5.

The maximum enhancement due to nuclei can be estimated assuming that there is no shadowing, and each
Table 1. Relative contributions to nuclear enhancement factor $k_{\text {nuc }}$.

\begin{tabular}{lll}
\hline & $\sigma \propto A_{1} A_{2}$ & $\sigma \propto A_{1}^{2 / 3} A_{2}^{2 / 3}$ \\
\hline $\mathrm{p}-\mathrm{H}$ & 1 & 1 \\
$\mathrm{p}-\mathrm{He}+\alpha-\mathrm{H}$ & 0.66 & 0.42 \\
$\alpha-\mathrm{He}$ & 0.11 & 0.043 \\
$\mathrm{CNO}$ & 0.11 & 0.042 \\
$\mathrm{NeMgSiS}$ & 0.014 & 0.0045 \\
$\mathrm{Fe}$ & 0.022 & 0.0042 \\
Total & 1.92 & 1.52 \\
\hline
\end{tabular}

nucleon participates independently in the scattering. On the other hand, an apparent minimum value of $k_{n u c}$ can be obtained by assuming a geometrical shadowing $\propto A^{2 / 3}$, as if the nucleons were tightly packed in the nucleus.

Table 1 shows the relative contributions of various nuclei or nucleon groups to $k_{\text {nuc }}$, under the two extremes that $\sigma \propto A_{1} A_{2}$ and $\sigma \propto A_{1}^{2 / 3} A_{2}^{2 / 3}$. The notation is such that the row labeled $\mathrm{CNO}$ includes all reactions of $\mathrm{C}, \mathrm{N}$, and $\mathrm{O}$ in the cosmic rays and in the ISM involving $\mathrm{p}, \mathrm{He}$, and $\alpha$ particles. Similarly, $\mathrm{NeMgSiS}$ includes all reactions of $\mathrm{CR}$ and ISM Ne, $\mathrm{Mg}, \mathrm{Si}$, and $\mathrm{S}$ in the cosmic rays and ISM that interact with $\mathrm{p}, \alpha$, and CNO. For this estimate, we assume the same cosmic ray as ISM composition for $\mathrm{NeMgSiS}$.

Note that reactions involving ions heavier that $\mathrm{He}$ account for up to $5 \%$ of the emissivity, so cannot be in any sense neglected. This estimate shows that $k_{n u c}=2.0$ exceeds the upper limit, so that the emissivity calculations in Fig. 2 are, if anything, an overestimation. Mori [32], using DPMJET-3 to derive cosmic-ray energy-dependent emissivities, does not give results below $6 \mathrm{GeV} /$ nuc. Recent treatments are given by [34] who, using different compositions, find $1.9 \lesssim k_{n u c} \lesssim 2.1$, in better agreement with the "no-shadowing" cross sections. As noted there, the situation is more complicated than a simple value of $k_{n u c}$, which is dependent on the photon energy and the differences between cosmic-ray proton and ion spectra. Other treatments find corrections $\propto A^{2.2 / 3}$ [35]. The low value of $k_{n u c}=1.45$ obtained in my early study [23] was a consequence of using a cross-section correction closer to the $A^{2 / 3}$ behavior, and by using a low value for the cosmicray $\alpha$ particle to proton ratio.

\subsection{Cosmic-ray spectral hardenings}

Cosmic-ray spectral hardenings at $\approx 300 \mathrm{GV}$ in the $\mathrm{p}$ and $\alpha$ spectra could be due to any number of physical effects. From general principles, we can suggest either that this spectral hardening is due to (1) the superposition of two populations with different mean injection indices; (2) rigidity-dependent effects on escape from the Galaxy; (3) rigidity-dependent effects at the source/accelerator; (4) acceleration during transport; or (5) grammage effects.

Effect (5) fails because grammage makes instead a low-energy hardening when heavier ( $\gtrsim \mathrm{CNO}$ ) low-energy cosmic-rays pass through significant grammages during escape. Effect (4), which suggests turbulent reacceleration, would make curved, not broken power-law spectra [36]. Effects (2) and (3) go hand-in-hand, in terms of the behavior if not in explanation. All Fermi acceleration 
theories have, in the collisionless limit, a dependence on rigidity as the fundamental quantity. A break or hardening at a given rigidity should manifest as one of the 3 (Bernard) Peters cycle predicting $E_{b r} \propto Z$, as described by Dr. Tilav at this workshop.

Idea (1) seems to be ruled out from a number of directions. A 0.2 break from the addition of two underlying distributions that only differ by 0.2 in index would be extremely broad, and there would be unusual changes in composition depending on the compositions of the 2 populations. Which returns us to (2) and (3), for which we need an explanation why, during cascading from small to large $k$, the wave turbulence spectrum governing diffusion would apparently soften, so that lower energy ions that gyroresonate with these waves would escape more readily than at high energies resulting in a hardening.

\section{6. $\bar{p} / p$ ratio}

The figure in the AMS-02 release [1] showing a severe disagreement of the $\bar{p} / p$ ratio with a secondary production model prediction deserves a few comments. First is that ratios $\bar{p} / p$ and $e^{+} /\left(e^{+}+e^{-}\right)$are far less useful than the particle spectral intensities themselves [37], particularly when looking at fits to data using models involving multiple source populations. Second is that the model [5] showing a discrepancy with the AMS-02 data, used to constrain fits for dark matter candidates to $\bar{p}$ production, was tailored to fit PAMELA data that becomes increasingly uncertain at high energies.

If there are $\bar{p} / p$ enhancements compared to expectations from secondary production, a non-Copernican explanation has us fortuitously situated some $2 \mathrm{Myr}$ ago next to a $\mathrm{SN}$ that deposited ${ }^{60} \mathrm{Fe}$ while enhancing cosmicray production in $\bar{p}$ and $\mathrm{e}^{+}$[38]. Fits to the $\bar{p} / p$ data with a conventional propagation parameters $(1 / 3<\delta \lesssim 1 / 2)$ in a secondary nuclear production models are found in recent analyses $[39,40]$. In particular, agreement with the $\mathrm{B} / \mathrm{C}$ and $\bar{p} / p$ ratio with a propagation model with $\delta \approx 0.42$ is found in [41].

\subsection{Secondary nuclear production models}

The high-quality Fermi-LAT data demands increasingly accurate nuclear data, which is currently lacking. Some new low-energy, $\lesssim 1 \mathrm{GeV} /$ nuc data are presented in [42] and were discussed at this workshop by Dr. Taylor. Another paper that would be a valuable benchmark for low-energy secondary production calculations is [43]. All the new data is at $T_{n u c} \lesssim 2 \mathrm{GeV} /$ nuc. The important regime for $\gamma$-ray astronomy is at $\approx 2-10 \mathrm{GeV} /$ nuc, where scaling models break down, event generators extrapolate outside their zone of certainty, and exclusive formulations become impossible.

Rather than providing empirical formulations that are specific, model-dependent, and can't be tampered with, a better direction is to have physical models that can be modified. Detailed examination of processes in the regime is required. Intermediate-energy nuclear astrophysics is not, unfortunately, a growth area.

A relevant formula to conclude this contribution is the $\pi^{0}$-production threshold kinetic energy per nucleon
$T_{t h r, n u c}$ of a cosmic-ray ion with mass $M=A_{1} m_{p}$ striking an interstellar atom or ion with mass $m=A_{0} m_{p}$. The threshold condition is that $s=E^{2}-p^{2}=(m+M+$ $\left.m_{\pi^{0}}\right)^{2}$. I obtain

$$
T_{t h r, n u c}=m_{\pi}\left(\frac{1}{A_{1}}+\frac{1}{A_{0}}+\frac{m_{\pi}}{2 A_{1} A_{0} m_{p}}\right) .
$$

Thus $T_{t h r, n u c}=2 m_{\pi}+m_{\pi}^{2} / 2 m_{p} \cong 280 \mathrm{MeV}$, as is well known. This also implies that $T_{t h r, n u c}=171 \mathrm{MeV} /$ nuc for $\mathrm{p}-\mathrm{He}$ and $\alpha$-p, and $T_{t h r, n u c}=69.9 \mathrm{MeV} /$ nuc for $\alpha-\mathrm{He}$ collisions. Concerns [42] about the accuracy of near-threshold $p+p \rightarrow \pi$ cross sections [44] are not as important as near-threshold contributions of cosmic-ray nuclei.

\section{Concluding remarks}

These few pages should not conceal the much more detailed and exhaustive work that must be done to satisfy cosmic-ray constraints. For this, one may consult the detailed GALPROP treatment [45], which requires processes not often studied, for example, nuclear energy losses from pion production [46], or semi-analytic treatments, e.g., [47].

The new AMS-02 confirms the hardening in the cosmic-ray proton and $\alpha$ spectrum reported with PAMELA, and the two data sets are typically within $\sim 5 \%$ of each other. With this accuracy, and the quality of the Fermi-LAT emissivity data, renewed focus on metallicity corrections and secondary production processes at the $\sim 5 \%$ level is required.

Before explaining the shape of the LISMCRp spectrum, we must be sure that we can determine its spectrum from as low an energy, $R \ll 1 \mathrm{GV}\left(E_{n u c} \lesssim\right.$ $100 \mathrm{MeV} /$ nuc), below which Solar energetic particles and anomalous cosmic rays start to dominate, to as high as energy as possible. It is generally believed that Solar modulation effects become negligible above $R \sim 10 \mathrm{GV}$. From this boundary condition, we should be able to track the $\gamma$-ray spectrum back to the cosmic rays and their sources. This is work in progress.

I thank Drs. Elisa Prandini, Simona Toscano, and Andrii Neronov for the kind invitation to visit Geneva and attend the workshop. Comments of M. Kachelriess, S. Ostapchenko, T. Kamae, and particularly A. Strong are much appreciated. Secondary nuclear production code used here is available by e-mail request: charles.dermer@nrl.navy.mil.

\section{References}

[1] http://www.ams02.org, "AMS Days at CERN" and Latest Results

[2] Aguilar, M., Alberti, G., Alpat, B., et al., Physical Review Letters 110, 141102 (2013)

[3] Aguilar, M., Aisa, D., Alvino, A., et al., Physical Review Letters 113, 121102 (2014)

[4] See http://www.mpe.mpg.de/ aws/propagate. html for cosmic-ray data and resources

[5] Donato, F., Maurin, D., Brun, P., Delahaye, T., \& Salati, P., Phys. Rev. Lett. 102071301 (2009) 
[6] 33rd Intern. Cosmic Ray Conf., 2013, Precision Measurement of the Cosmic Ray Boronto-Carbon Ratio with AMS [AMS collaboration], ed. P. 33rd Intern. Cosmic Ray Conf. See also http: //www . ams02.org/, New Results from AMS Presented at ICRC 2013

[7] O. Adriani, et al., Science 332, 69 (2011)

[8] Adriani, O., Barbarino, G.C., Bazilevskaya, G.A., et al. Phys. Reports 544, 323 (2014)

[9] Dermer, C.D., Finke, J.D., Murphy, R.J., et al. 2012 Fermi Symposium proceedings - eConf C121028, arXiv:1303.6482 (2013)

[10] Dermer, C.D., Strong, A.W., Orlando, E., Tibaldo, L., \& for the Fermi Collaboration, arXiv:1307.0497, 2013 ICRC conference (2013)

[11] A.D. Panov, et al., arXiv:astro-ph/0612377 (2006)

[12] Stone, E.C., Cummings, A.C., McDonald, F.B., et al. Science 341, 150 (2013)

[13] Kachelrieß, M., \& Ostapchenko, S. Phys. Rev. D 86, 043004 (2012)

[14] Blasi, P. Astron. Astrophys. Rev. 21, 70 (2013)

[15] Ackermann, M., Ajello, M., Allafort, A., et al. Science, 339, 807 (2013)

[16] Aharonian, F., Akhperjanian, A.G., Bazer-Bachi, A.R., et al. Astron. Astrophys. 464, 235 (2007)

[17] Blandford, R., \& Eichler, D. Phys. Rep. 154, 1 (1987)

[18] Dermer, C.D. Physical Review Letters, 109, 091101 (2012)

[19] Strong, A.W., Moskalenko, I.V., \& Ptuskin, V.S., Ann. Rev. Nuclear and Particle Sci. 57, 285 (2007)

[20] A. Neronov, D.V. Semikoz, and A.M. Taylor, Phys. Rev. Lett. 108, 051105 (2012)

[21] Genolini, Y., Putze, A., Salati, P., \& Serpico, P.D. 2015, arXiv: 1504.03134

[22] Adriani, O., Barbarino, G.C., Bazilevskaya, G.A., et al., Astrophys. J. 791, 93 (2014)

[23] Dermer, C.D., Astron. \& Astrophys. 157, 223 (1986)

[24] Simpson, J.A., Ann. Rev. Nuclear and Particle Science 33, 323 (1983)

[25] A.A. Abdo, et al., Astrophys. J. 703, 1249 (2009)
[26] Casandjian, J.-M., "Diffuse galactic radiation," American Institute of Physics Conference Series 1505, 37 (2012)

[27] Kamae, T., Karlsson, N., Mizuno, T., Abe, T., \& Koi, T., Astrophys. J. 647, 692 (2006)

[28] Orlando, E., \& Strong, A., MNRAS 436, 2127 (2013)

[29] Casandjian, J.-M., submitted (2015)

[30] Strong, A. W., et al., in preparation (2015)

[31] Gaggero, D., Grasso, D., Marinelli, A., Urbano, A., \& Valli, M., arXiv:1504.00227 (2015)

[32] Mori, M. 2009, Astroparticle Physics 31, 341

[33] Meyer, J.-P., Astrophys. J. Supp. 57, 173 (1985)

[34] Kachelriess, M., Moskalenko, I.V., \& Ostapchenko, S. S., Astrophys. J. 789, 136 (2014)

[35] Norbury, J.W., \& Townsend, L.W. Nuclear Instruments and Methods in Physics Research B 254, 187 (2007)

[36] Becker, P.A., Le, T., \& Dermer, C.D., Astrophys. J. 647, 539 (2006)

[37] Kappl, R., \& Winkler, M.W., J. Cosmology Astrophys. 9, 051 (2014)

[38] Kachelriess, M., Moskalenko, I.V., \& Ostapchenko, S. S. arXiv:1502.04158 (2015)

[39] Lin, S.-J., Bi, X.-J., Yin, P.-F., \& Yu, Z.-H. 2015, arXiv: 1504.07230 (2015)

[40] Giesen, G., Boudaud, M., Genolini, Y., et al., arXiv: 1504.04276 (2015)

[41] Evoli, C., Gaggero, D., \& Grasso, D., arXiv: 1504.05175 (2015)

[42] Kafexhiu, E., Aharonian, F., Taylor, A.M., \& Vila, G.S. Phys. Rev. D 90, 123014 (2014)

[43] Abaev, V.V., Fedorova-Koval, E.P., Gridnev, A.B., et al., J. Phys. G Nuclear Physics 14, 903 (1988)

[44] Dermer, C.D. Astrophys. J. 307, 47 (1986)

[45] Moskalenko, I.V., Strong, A.W., Ormes, J.F., \& Potgieter, M.S., Astrophys. J. 565, 280 (2002)

[46] Krakau, S., \& Schlickeiser, R., Astrophys. J. 802, 114 (2015)

[47] Blasi, P., \& Amato, E., J. Cosmology Astrophys. 1, 010 (2012) 